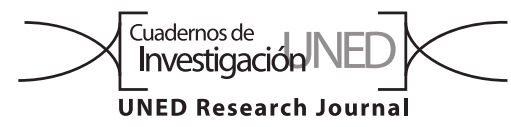

\title{
Maíces nativos de Guanacaste, Costa Rica: caracterización de los granos
}

\author{
Patricia Oreamuno-Fonseca' \& José Eladio Monge-Pérez ${ }^{2}$ \\ 1. Escuela de Ciencias Exactas y Naturales, Universidad Estatal a Distancia, San José, Costa Rica; patrioref@gmail.com \\ 2. Estación Experimental Agrícola Fabio Baudrit Moreno y Sede de Guanacaste, Universidad de Costa Rica, Costa Rica; \\ jose.mongeperez@ucr.ac.cr
}

Recibido 23-I-2018 • Corregido 10-III-2018 • Aceptado 30-IV-2018

\begin{abstract}
Maize landraces of Guanacaste, Costa Rica: grain characterization. There are no reports addressing the characterization of grains of maize landraces from Costa Rica. From April to August 2013, we collected grain samples of maize (Zea mays L.) landraces (also called creole maize) in Nicoya and Hojancha cantons, Guanacaste province, Costa Rica, through visits to different local growers. We characterized grains of five maize landraces (Amarillo, Congo, Piquinitillo, Pujagua and Rocarina), considering both qualitative (three) and quantitative (seven) variables. There were statistically significant differences between phenotypes for quantitative variables. Data show a wide variability with respect to grain length $(9,10-11,96 \mathrm{~mm})$, grain width $(6,41$ $-9,12 \mathrm{~mm})$, grain thickness $(3,27-4,49 \mathrm{~mm})$, grain length/width ratio $(1,17-1,77)$, grain length/thickness ratio $(2,24-3,69)$, grain width/ thickness ratio $(1,71-2,39)$, and weight of 100 grains $(16,80-30,60 \mathrm{~g})$. This is the first report about the existence of Piquinitillo and Rocarina landraces in Costa Rica, as well as the first report about grain characterization of maize landraces from Costa Rica.
\end{abstract}

KEY WORDS: maize, Zea mays, phenotypes, creole varieties, landraces.

El maíz es el aporte más importante de los pueblos de Mesoamérica a la humanidad; actualmente es el tercer cultivo más sembrado a nivel mundial, luego del trigo y el arroz (Ávila, Morales, \& Ortega, 2016). El centro de origen y domesticación del maíz se extiende a lo largo de México y hasta Guatemala. Para Centroamérica (excepto Guatemala) y Panamá existen 11 razas de maíz; y para el continente americano unas 300 razas (Serratos, 2009).

Las comunidades rurales han generado muchas variantes de maíz, para darles diferentes usos; los maíces morados se utilizan para ser consumidos en elotes, atoles, pozole y tortillas; los amarillos se usan como forraje para animales; y con el maíz blanco y otras variantes se elaboran tamales, tortillas, atoles, elotes cocidos y esquites (Ávila, Morales \& Ortega, 2016).
RESUMEN: No existe información sobre caracterización de los granos de maíces nativos de Costa Rica. De abril a agosto de 2013, recolectamos muestras de granos de maíces (Zea mays L.) nativos (también llamados criollos) presentes en los cantones de Nicoya y Hojancha, de la provincia de Guanacaste, Costa Rica, mediante visitas a diversos agricultores de esa zona. Hicimos una caracterización de los granos de cinco maíces nativos (Amarillo, Congo, Piquinitillo, Pujagua y Rocarina), tanto a nivel cualitativo (tres variables) como cuantitativo (siete variables). Se presentaron diferencias estadísticamente significativas entre fenotipos en el caso de las variables cuantitativas. Los datos mostraron una amplia variabilidad en cuanto a la longitud del grano $(9,10-11,96$ $\mathrm{mm})$, ancho del grano $(6,41-9,12 \mathrm{~mm})$, grosor del grano $(3,27-4,49$ $\mathrm{mm})$, índice longitud/ancho del grano $(1,17-1,77)$, índice longitud/ grosor del grano $(2,24-3,69)$, índice ancho/grosor del grano $(1,71-$ $2,39)$, y peso de 100 granos ( $16,80-30,60 \mathrm{~g}$ ). Esta es la primera vez que se informa sobre la existencia de los maíces Piquinitillo y Rocarina en Costa Rica, y también la primera vez que se informa sobre la caracterización de los granos de maíces nativos de Costa Rica.

PALABRAS CLAVE: maíz, Zea mays, fenotipos, variedades criollas, variedades nativas.

En México, los maíces nativos tienen un arraigo ancestral en la vida de la población; en 2014, el 65\% de la superficie cultivada con maíz en ese país se sembró con maíces nativos; entre las 60 razas descritas de maíces nativos de ese país se encuentran variedades de color blanco, azul, negro, rojo, morado, rosa, crema, amarillo y anaranjado (Uriarte-Aceves et al., 2015; López-Torres, Rendón-Medel \& Camacho, 2016).

Los maíces nativos poseen una gran diversidad genética y potencial para producir alimentos funcionales que ayuden a enfrentar problemas de salud; contienen compuestos nutracéuticos, como fibra dietética, compuestos fenólicos, carotenoides, y ácidos grasos omega 6 (Serna-Saldívar, Gutiérrez-Uribe, Mora-Rochín \& García-Lara, 2013). Estos compuestos ayudan a bloquear 
el crecimiento de tumores, y a reducir el riesgo de enfermedades cardiovasculares (Uarrota, Severino \& Maraschin, 2011). Los maíces de color azul y morado, por su alto contenido de antocianinas, poseen un gran efecto antioxidante, anticancerígeno y antimutagénico, y ayudan a reducir los problemas de hipertensión arterial (Castañeda-Sánchez, 2011; Ronceros et al., 2012; SalinasMoreno, Pérez-Alonso, Vázquez-Carrillo, Aragón-Cuevas \& Velázquez-Cardelas, 2012; Guillén-Sánchez, MoriArismendi \& Paucar-Menacho, 2014; Uriarte-Aceves et al., 2015; Hernández-Martínez etal., 2016).

En diversos países, los campesinos utilizan el término "maíz criollo" para denotar un material nativo de la comunidad, región, estado o país, y que se diferencia de un material extranjero, un maíz híbrido o una variedad mejorada. Son materiales que han sido formados por los agricultores durante años, mediante selección empírica, y los conservan y manejan año tras año en un complejo sistema de intercambio de semillas y genes (Acosta, 2009; Sánchez-Hernández, Cruz-Lázaro \& Sánchez-Hernández, 2015).

Los maíces criollos son poblaciones heterogéneas, homo-heterocigotas, desarrolladas y conservadas por agricultores a través de muchas generaciones de selección para caracteres específicos, como textura de grano, color, forma del grano, forma de mazorca, entre otros. El término "criollo" también se usa para identificar a las poblaciones segregantes, producto de cruzamientos entre materiales nativos con híbridos o variedades mejoradas, siempre que el producto final mantenga una mayor proporción de caracteres de interés para los agricultores. Dado que el maíz es una planta originaria de Mesoamérica, se sugiere que el término más adecuado para denominar el recurso fitogenético es "maíz nativo", en vez de "maíz criollo" (Acosta, 2009; SánchezHernández et al., 2015).

Una variedad nativa se define como: "población dinámica de una planta cultivada que tiene origen histórico, identidad propia, que no se deriva de un proceso de mejoramiento genético formal, y que a menudo presenta diversidad genética, adaptación a condiciones locales, y asociación con sistemas tradicionales de cultivo". Por otra parte, una variedad criolla es: "aquella que se derivó a partir de una variedad originalmente mejorada, la cual luego se convirtió en una variedad tradicional a través de numerosos ciclos de siembra y selección de semilla por los agricultores, en un sitio específico". Generalmente se considera que se requiere cultivar una variedad por un período de entre 30 a 100 años para que se pueda considerar como una variedad nativa (Camacho, Maxted, Scholten \& Ford-Lloyd, 2006).
Algunas ventajas de los maíces nativos son: mejor manejo del riesgo agrícola, adaptación a las condiciones climáticas locales, estabilidad a la variabilidad climática, resistencia a plagas y enfermedades, costos de producción más bajos, y aptitud para la elaboración de preparaciones culinarias tradicionales (López-Romero et al., 2005; Camacho et al., 2006; Olson, Morris \& Méndez, 2012; Cortés, Hernández, Sangerman-Jarquín \& Morán, 2013; Fernández, Morales \& Gálvez, 2013). Las variedades nativas de maíz muchas veces muestran un comportamiento agronómico igual o superior al de las variedades mejoradas (Sandri \& Tofanelli, 2008; Olson et al., 2012; Junior, Melo, Matias \& Fontes, 2015). Los productores confían en los maíces criollos por ser opciones resistentes y predecibles, por lo que pueden ser una herramienta importante para hacer frente al cambio climático (Hellin, Keleman, López, Donnet \& Flores, 2013).

En Costa Rica se tienen registros del cultivo de maíz en la zona de Nicoya desde el año 1000 (Sibaja \& Zelaya, 2015). En 1526, Gonzalo Fernández de Oviedo describió el sistema de siembra de maíz en Nicoya, e indicó que se recogían cosechas tres veces al año; el maíz se consumía como tortillas, pinolillo, chicha, entre otros. En 1548, los nativos de Nicoya debían pagar como tributo indígena a la corona española un total de 4500 fanegas de maíz por año, además de frijoles, miel, cera, sal, gallinas, tejidos y objetos de cerámica (Ibarra, 2003).

En el pasado se han realizado esfuerzos por colectar y caracterizar los maíces nativos de Costa Rica (Brenes, 2013). En 1989, existían 393 introducciones de este país en el Banco de Germoplasma del Centro Internacional para el Mejoramiento de Maíz y Trigo (CIMMYT). Entre 1989 y 1993 se recolectaron 60 introducciones de variedades locales de maíz, y se recopiló información de algunas características de las mismas, y de las prácticas de cultivo asociadas a ellas (Brenes, Saborío, Madriz, Campos \& Bolaños, 1993). Sin embargo, en dicho trabajo no se realizó la caracterización morfológica de esas variedades.

En 2014, el Gobierno de Costa Rica indicó que existían entre 80 a 100 variedades de maíz criollo en el país, por lo que declaró como Patrimonio Cultural el maíz en sus variedades nativas y criollas, y las tradiciones, prácticas agro-culturales, usos, saberes, sabores y colores asociados al grano. Asimismo, indicó que en Guanacaste existe una rica y variada gastronomía basada en el uso del maíz (Ministerio de Cultura y Juventud, 2014).

Un factor esencial para la adecuada conservación y mejoramiento de los maíces nativos es conocer sus características, y poder identificarlos en campo de manera sencilla (Ávila et al., 2016). El objetivo del presente trabajo fue realizar una caracterización de los granos de 
algunos de los maíces nativos presentes en Guanacaste, Costa Rica.

\section{MATERIALES Y MÉTODOS}

En los meses de abril a agosto de 2013, se recolectaron muestras de granos de maíces (Zea mays L.) nativos (también llamados criollos) presentes en los cantones de Nicoya y Hojancha, de la provincia de Guanacaste, Costa Rica, mediante visitas a diversos agricultores de esa zona.

Según la lista de descriptores de maíz (IBPGR, 1991), se seleccionaron para evaluación tres variables cualitativas (color del grano, tipo de grano, y forma de la superficie del grano) y siete variables cuantitativas: longitud, ancho y grosor del grano (en milímetros), índice longitud/ancho del grano, índice longitud/grosor del grano, índice ancho/grosor del grano, y peso de 100 granos (en gramos). Las mediciones se hicieron en un total de 10 granos por variedad, excepto para la variable peso de 100 granos, para lo cual se evaluaron cinco muestras de 100 granos cada una. Para la evaluación de las dimensiones de los granos se utilizó un calibrador digital milimétrico marca Mitutoyo, modelo $C D$, con una capacidad de $15,00 \pm 0,01 \mathrm{~cm}$, y para el peso de los granos se utilizó una balanza electrónica marca Ocony, modelo TH-I-EK, con una capacidad de $5000,0 \pm 0,1 \mathrm{~g}$.

Para las variables cuantitativas, se realizó un análisis de varianza, y se utilizó la prueba de LSD Fisher $(p \leq 0,05)$ para la comparación entre tratamientos.

\section{RESULTADOS}

Se colectaron muestras de granos de los siguientes maíces nativos: Piquinitillo; Pujagua ( 2 accesiones); Rocarina (también llamado Blanco Rosarina), Congo, y Amarillo (Fig. 1). De estos fenotipos, solamente se tienen referencias a nivel de caracterización de los granos para las variedades Amarillo y Pujagua, a partir de investigaciones realizadas en Nicaragua (Morales, 1993; López,
1997; Carrasco \& Pineda, 2009; Melgara \& Tinoco, 2013; Mendoza \& Gaitán, 2013). Sin embargo, no es posible asegurar que se trate exactamente de los mismos fenotipos, sino que probablemente se trata de un nombre genérico por el color del grano en el caso de la variedad Amarillo; $y$ en el caso del Pujagua, según la Real Academia Española, la palabra "pujagua" significa en Nicaragua "variedad de maíz de mazorca pequeña con grano morado" (Real Academia Española, 2017).

A nivel de Costa Rica, solamente se tienen informes anteriores sobre la presencia de la variedad Pujagua en la provincia de Guanacaste, específicamente en las localidades de Monte Romo de Hojancha, y Curubandé de Liberia, y de la variedad Amarillo en las localidades de Tierra Blanca y San Rafael de Oreamuno, en la provincia de Cartago. Con respecto al maíz Pujagua, se indica que en Monte Romo es un "maíz morado especialmente usado para preparar una bebida fermentada conocida como chicheme", y que en Curubandé es "un maíz color púrpura, muy gustado para la preparación de tortilla dulce (repostería de masa de maíz, queso y azúcar), atoles y chicha (licor fermentado)" (Brenes et al., 1993).

Características cualitativas: Los maíces Congo y Pujagua presentaron granos de color morado, de tipo cristalino y con superficie redonda (mayoritariamente) (Cuadro 1); los granos del Congo tienen una coloración más oscura que los del Pujagua (Fig. 1). Los otros maíces nativos mostraron granos de tipo dentado. La variedad Rocarina fue la única que presentó granos de color blanco, mientras que Piquinitillo mostró granos de color amarillo, y la variedad Amarillo presentó granos de color anaranjado.

Longitud del grano: La variedad Rocarina presentó los granos más largos, mientras que la variedad Congo mostró los granos más cortos (Fig. 2). No hubo diferencias significativas entre las dos accesiones de maíz Pujagua.

En el caso del maíz Amarillo, otros investigadores han encontrado valores entre 9,70 y $11,15 \mathrm{~mm}$ para esta

CUADRO 1

Características cualitativas de los granos de los maíces nativos

\begin{tabular}{cccc} 
Variedad & Color del grano & Tipo de grano & Forma de la superficie del grano \\
Amarillo & Anaranjado & Dentado & Dentado \\
Congo & Morado oscuro & Cristalino & Redondo \\
Piquinitillo & Amarillo & Dentado & Dentado \\
Pujagua & Morado & Cristalino & Redondo; algunos Plano \\
Rocarina & Blanco & Dentado & Dentado \\
\hline
\end{tabular}




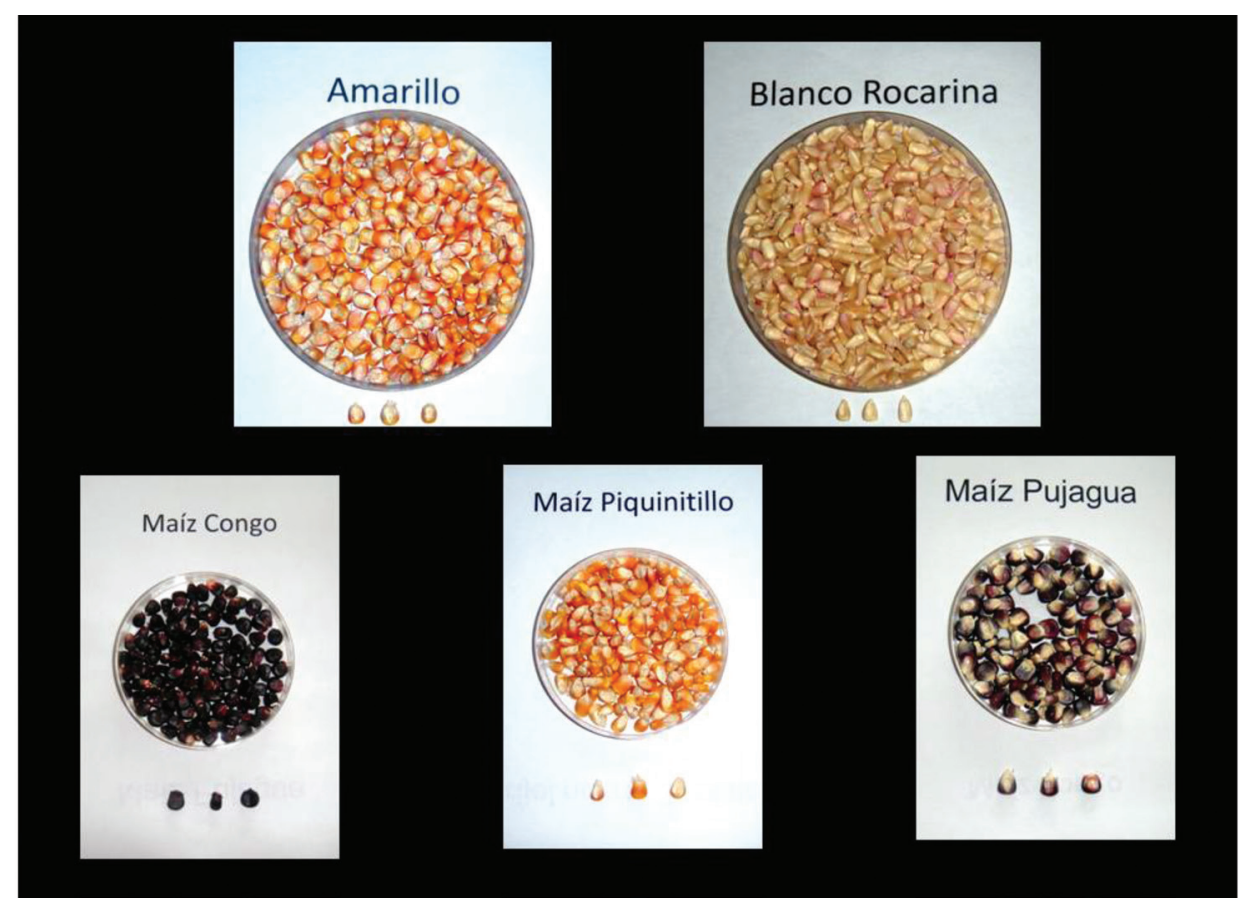

Fig. 1. Granos de los maíces nativos recolectados en Guanacaste.

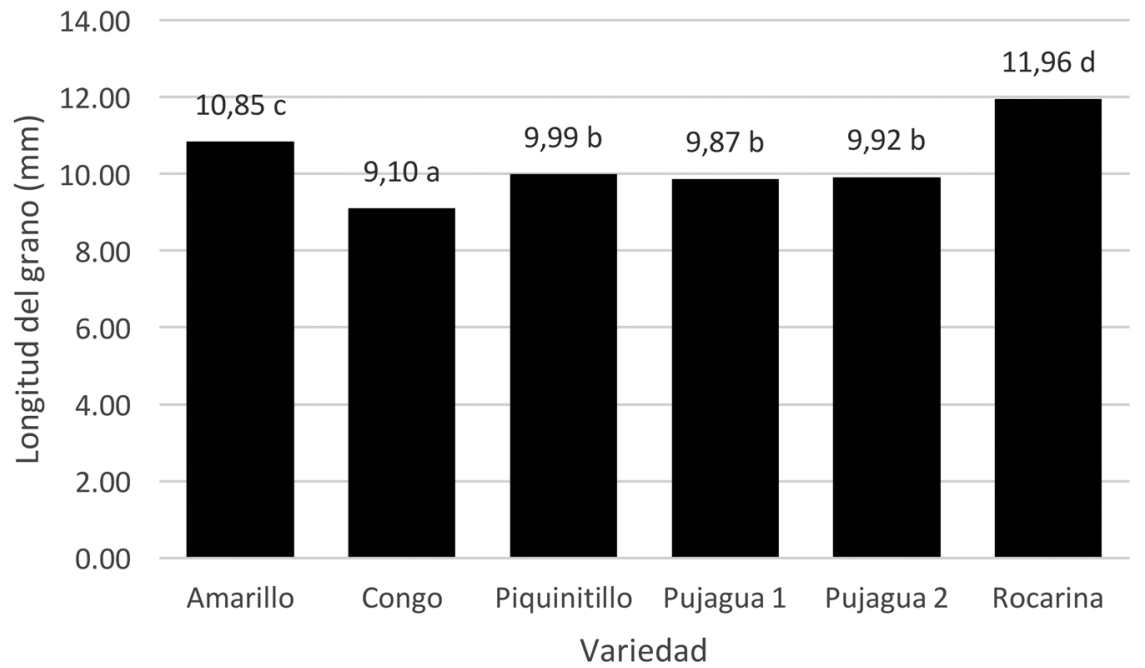

Fig. 2. Longitud del grano ( $\mathrm{mm}$ ) para los maíces nativos de Guanacaste. Medias con una letra en común no son significativamente diferentes entre sí, según la prueba LSD Fisher $(p \leq 0,05)$.

característica, mientras que para el maíz Pujagua los valores obtenidos han sido entre 7,79 y 10,91 mm (Morales, 1993; López, 1997; Carrasco \& Pineda, 2009; Melgara \& Tinoco, 2013; Mendoza \& Gaitán, 2013); los valores hallados en el presente trabajo se ubicaron dentro de dichos rangos.

Ancho del grano: Las variedades Congo, Piquinitillo y Rocarina mostraron los granos más angostos, mientras que la variedad Amarillo presentó los granos más anchos (Fig. 3). No hubo diferencias significativas entre las dos accesiones de maíz Pujagua.

Otros investigadores han hallado en el caso del maíz Amarillo, un ancho de grano entre 7,72 y $9,50 \mathrm{~mm}$, y para el maíz Pujagua entre 8,28 y 10,79mm (Morales, 1993; López, 1997; Carrasco \& Pineda, 2009; Melgara \& Tinoco, 2013; Mendoza \& Gaitán, 2013); los valores obtenidos en la presente investigación se ubicaron dentro de dichos 


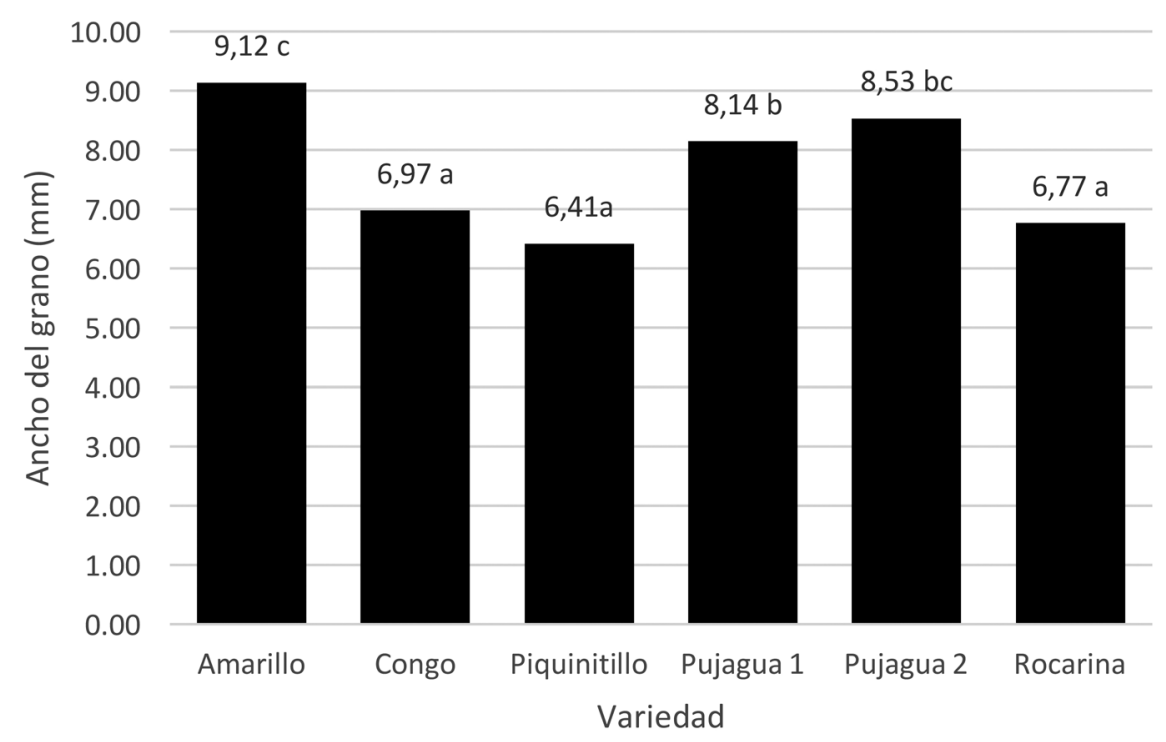

Fig. 3. Ancho del grano $(\mathrm{mm})$ para los maíces nativos de Guanacaste. Medias con una letra en común no son significativamente diferentes entre sí, según la prueba LSD Fisher $(p \leq 0,05)$.

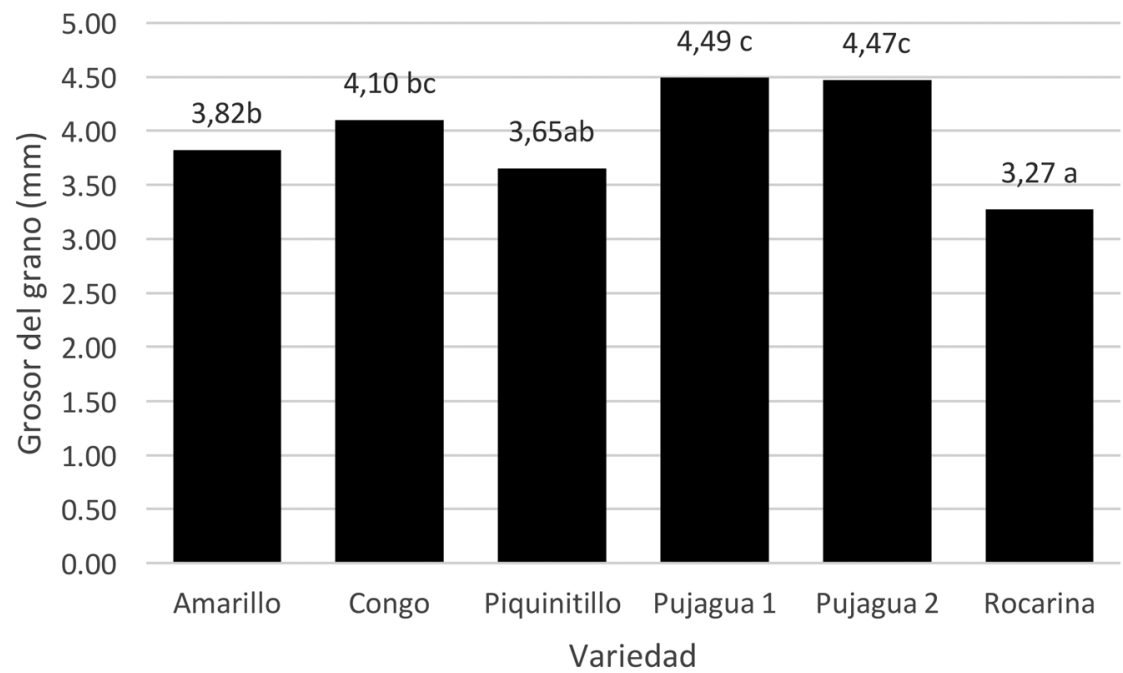

Fig. 4. Grosor del grano $(\mathrm{mm})$ para los maíces nativos de Guanacaste. Medias con una letra en común no son significativamente diferentes entre sí, según la prueba LSD Fisher $(p \leq 0,05)$.

rangos para el maíz Amarillo y el Pujagua 2, pero el maíz Pujagua 1 presentó un valor menor al mismo $(8,14 \mathrm{~mm})$.

Grosor del grano: La variedad Rocarina presentó los granos con el menor grosor, mientras que los granos más gruesos se obtuvieron en la variedad Pujagua (Fig. 4). No hubo diferencias significativas entre las dos accesiones de maíz Pujagua.

En otros trabajos, el grosor del grano se ha ubicado entre 3,50 y 5,04mm para el maíz Amarillo, y entre 3,67 y 5,10mm para el maíz Pujagua (Morales, 1993; López, 1997; Carrasco \& Pineda, 2009; Melgara \& Tinoco, 2013; Mendoza \& Gaitán, 2013); los valores obtenidos en el presente trabajo se ubicaron dentro de dichos rangos.

Índice longitud/ancho del grano: El mayor índice longitud/ancho del grano lo obtuvo el maíz Rocarina (forma más alargada), mientras que el menor valor para esta característica correspondió a los maíces Amarillo y Pujagua (forma menos alargada) (Fig. 5). En todo caso, 


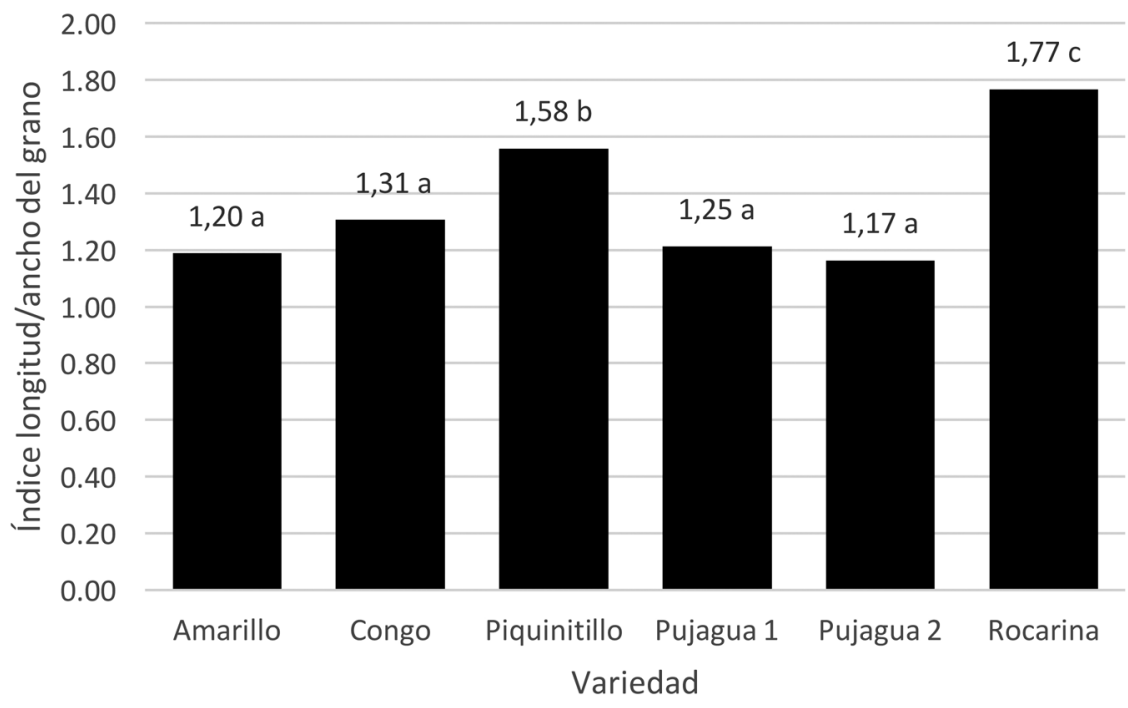

Fig. 5. Índice longitud/ancho del grano para los maíces nativos de Guanacaste. Medias con una letra en común no son significativamente diferentes entre sí, según la prueba LSD Fisher $(p \leq 0,05)$.

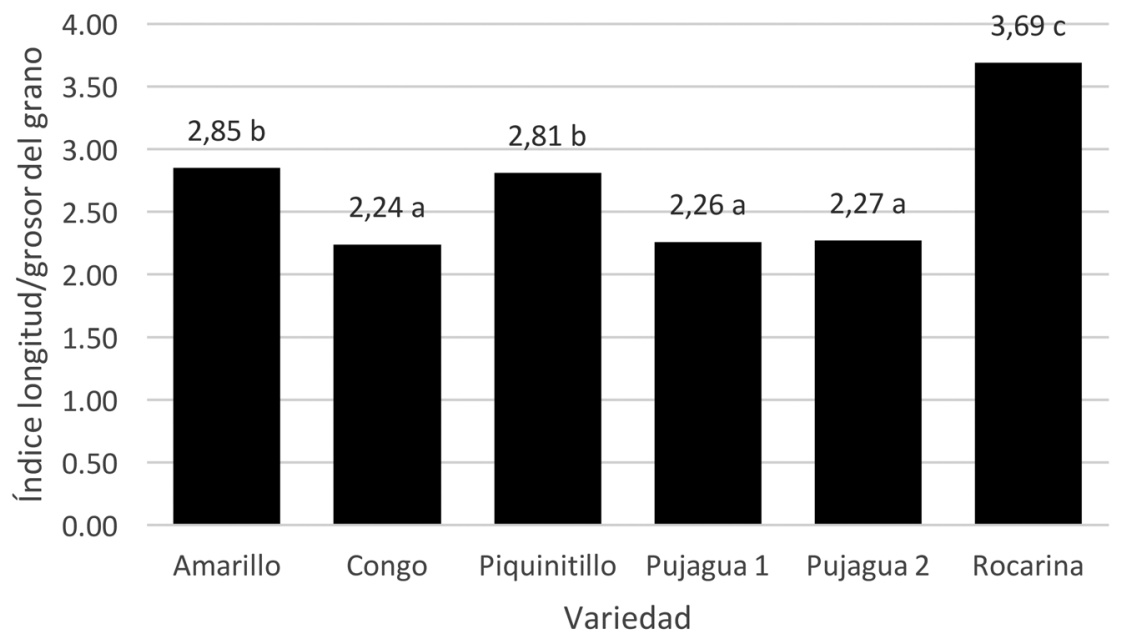

Fig. 6. Índice longitud/grosor del grano para los maíces nativos de Guanacaste. Medias con una letra en común no son significativamente diferentes entre sí, según la prueba LSD Fisher $(\mathrm{p} \leq 0,05)$.

todas las variedades obtuvieron valores superiores a 1,0 para esta variable, lo que indica que todas tienen granos de forma alargada.

Índice longitud/grosor del grano: Los granos con el mayor índice longitud/grosor correspondieron a la variedad Rocarina, mientras que los menores valores para esta característica correspondieron a las variedades Congo, Pujagua 1 y Pujagua 2 (todas de color morado) (Fig. 6).

Índice ancho/grosor del grano: El mayor valor para el índice ancho/grosor del grano lo obtuvo la variedad Amarillo, y el menor valor fue para la variedad Congo; las demás variedades mostraron valores intermedios (Fig. 7).
Peso de $\mathbf{1 0 0}$ granos: La variedad con los granos menos pesados fue Piquinitillo, y la variedad con los granos más pesados fue Amarillo (Fig. 8). En este caso, sí se presentaron diferencias significativas entre las dos accesiones de maíz Pujagua.

Medias con una letra en común no son significativamente diferentes entre sí, según la prueba LSD Fisher $(p \leq 0,05)$.

En evaluaciones anteriores, en el caso del maíz Amarillo se han encontrado valores entre 22,52 y 33,50 g para esta característica, mientras que para el maíz Pujagua los valores obtenidos han sido entre 21,78 y 


$$
3.00
$$

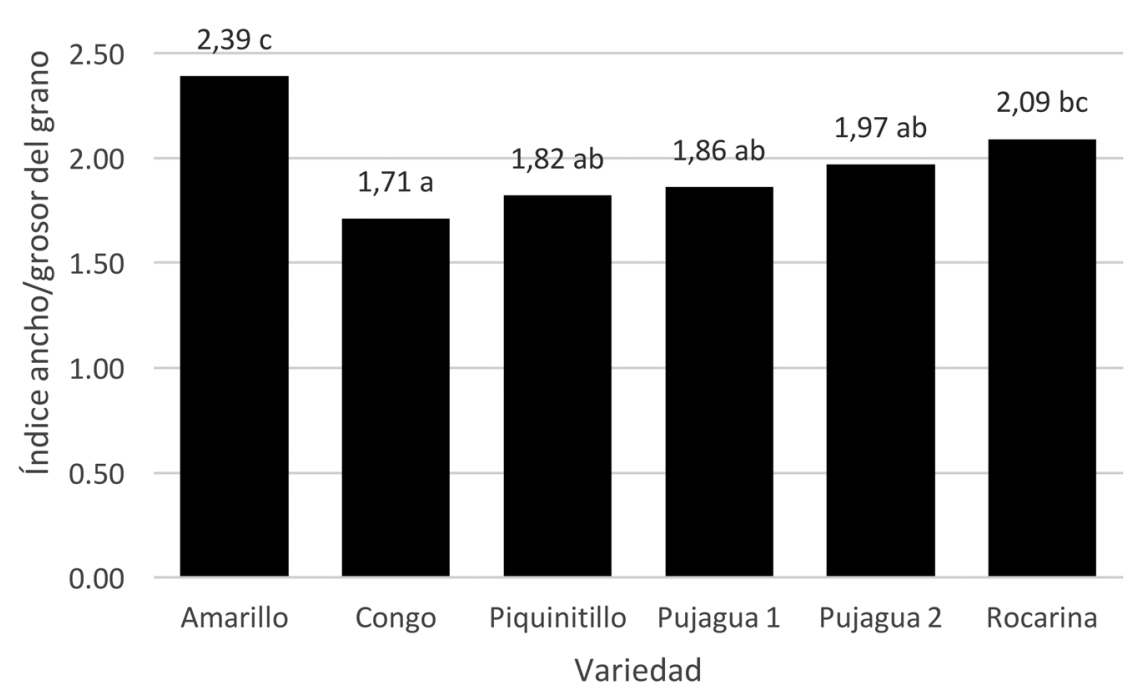

Fig. 7. Índice ancho/grosor del grano para los maíces nativos de Guanacaste. Medias con una letra en común no son significativamente diferentes entre sí, según la prueba LSD Fisher $(\mathrm{p} \leq 0,05)$.

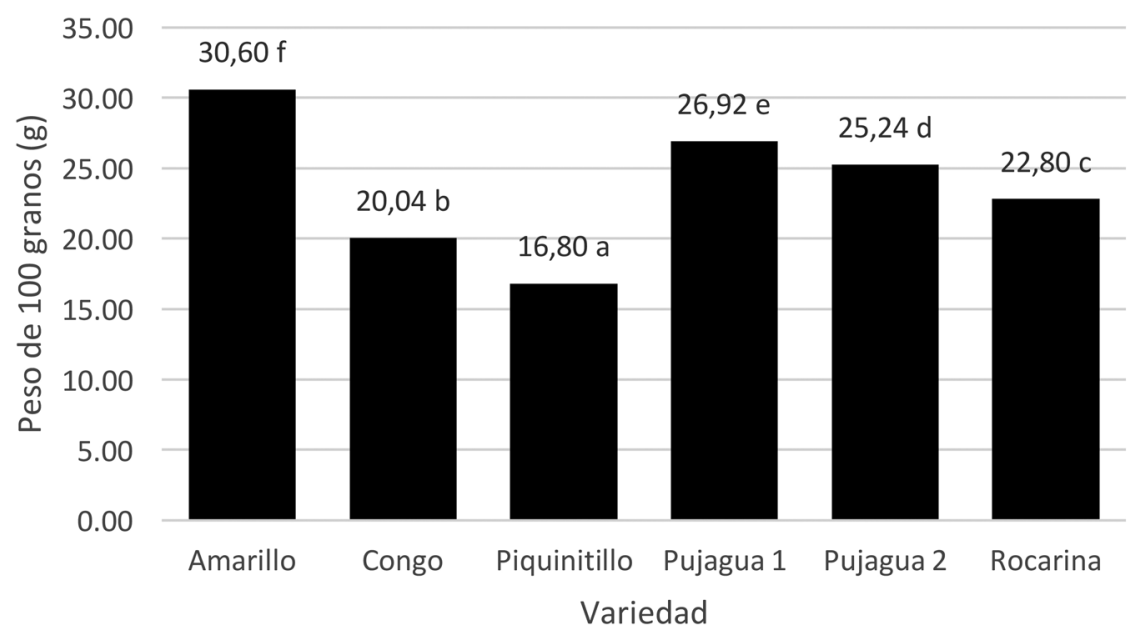

Fig. 8. Peso de 100 granos (g) para los maíces nativos de Guanacaste.

39,06 g (Morales, 1993; López, 1997; Carrasco \& Pineda, 2009; Melgara \& Tinoco, 2013; Mendoza \& Gaitán, 2013); los valores hallados en el presente ensayo se ubicaron dentro de dichos rangos.

\section{DISCUSIÓN}

Los maíces Pujagua y Congo se consideran entre las variedades de maíz nativo más comunes en Costa Rica, y específicamente en Guanacaste (Montoya, 2011; Ministerio de Cultura y Juventud, 2014). Ambos poseen granos de color morado, de tipo cristalino; esto indica que poseen un endospermo vítreo duro, cristalino y translúcido, con almidón en su mayoría córneo (Acosta, 2009).

Los maíces Amarillo, Piquinitillo y Rocarina, presentan granos de tipo dentado; esto indica que tienen un endospermo formado con almidón córneo cristalino, tanto en su exterior como interior, y que están coronados en la parte superior con almidón blando suave, que a la madurez origina una depresión central superior, debido a una mayor hidratación, dándole al grano la forma característica de diente (Acosta, 2009). 
Con respecto a la variedad Amarillo, aunque se tienen informes de dicha variedad en la zona de Cartago (Brenes et al., 1993), se considera poco probable que el maíz Amarillo presente en Guanacaste corresponda al mismo fenotipo, dadas las condiciones climáticas y edáficas tan diferentes entre ambas zonas del país; el nombre genérico de "amarillo", utilizado para una variedad de maíz, puede resultar confuso.

El maíz Amarillo presentó granos con una mayor longitud en relación al ancho, lo que hace que los mismos tengan una forma "alargada"; esto mismo se ha presentado en otras investigaciones con fenotipos que se denominan "Amarillo" en Nicaragua (Morales, 1993; López, 1997; Carrasco \& Pineda, 2009; Melgara \& Tinoco, 2013; Mendoza \& Gaitán, 2013). Este mismo comportamiento se observó en las dos accesiones de maíz Pujagua incluidas en el presente trabajo; sin embargo, otros investigadores en Nicaragua obtuvieron el mismo resultado con cinco accesiones de maíz Pujagua, pero hallaron el resultado contrario con otras diez accesiones de este tipo de maíz, lo que hizo que dichos granos presentaran forma "achatada" (valor del índice longitud/ancho del grano menor a 1,0) (Morales, 1993; López, 1997; Melgara \& Tinoco, 2013; Mendoza \& Gaitán, 2013).

En el caso de los maíces de color azul/morado, se ha informado sobre una alta correlación negativa entre el peso de 100 granos y el contenido de antocianinas totales, el contenido de fenoles solubles totales, y la actividad antioxidante, lo cual se explica por la ubicación del pigmento en las capas periféricas del grano (capa de aleurona); así, un grano pequeño tiene proporcionalmente más área pigmentada que un grano grande, por unidad de peso. Por lo tanto, en este tipo de maíces, es factible utilizar el color del grano y el peso de 100 granos como criterio de selección en un programa de mejoramiento genético en que interese el contenido de antocianinas totales (Salinas-Moreno et al., 2012); este puede ser el caso del maíz Congo, que presentó un menor peso de 100 granos y un color morado más intenso, en comparación con el Pujagua.

Es importante la conservación in situ del germoplasma adaptado a las condiciones ecofisiológicas del trópico seco mesoamericano, pues puede proveer información genética muy valiosa para los fitomejoradores y agricultores, con el fin de enfrentar las consecuencias del cambio climático.

Esta es la primera investigación en que se informa sobre la existencia de las variedades nativas de maíz denominadas Piquinitillo y Rocarina en Costa Rica, lo que se considera un hallazgo. Asimismo, este es el primer informe en que se documenta en forma accesible sobre la caracterización morfológica de los granos de maíces nativos de Costa Rica.

\section{AGRADECIMIENTOS}

Los autores agradecen el financiamiento recibido por parte de CSUCA/PRESANCAll, así como de la Universidad de Costa Rica, para la realización de este trabajo. Asimismo, agradecen la colaboración de Vania Solano, Susana Campo y Teresa Franco en el trabajo de campo, y de Mario Monge en la revisión de la traducción del resumen al idioma inglés.

\section{REFERENCIAS}

Acosta, R. (2009). El cultivo del maíz, su origen y clasificación; el maíz en Cuba. Cultivos Tropicales, 30(2), 113-120.

Ávila, C. H., Morales, J. A., \& Ortega, R. (2016). Los maíces nativos de la Sierra de Santa Marta; guía para su identificación en campo. Xalapa, Veracruz, México: Editorial Universidad Veracruzana.

Brenes, A. J. (26 de Enero de 2013). Conservación y potencial de maíces criollos en Costa Rica. La Nación (Costa Rica), pág. 27A.

Brenes, A., Saborío, J. C., Madriz, J., Campos, R., \& Bolaños, H. (1993). Recolección de germoplasma de maíz en Costa Rica, con énfasis en zonas de altura. Heredia, Costa Rica: Programa de Recursos Fitogenéticos, Escuela de Ciencias Agrarias, Facultad de Ciencias de la Tierra y el Mar, Universidad Nacional.

Camacho, T. C., Maxted, N., Scholten, M., \& Ford-Lloyd, B. (2006). Defining and identifying crop landraces. Plant Genetic Resources, 3(3), 373-384.

Carrasco, L. C., \& Pineda, L. S. (2009). Evaluación de ocho genotipos de maíz (Zea mays L.) de polinización libre y tres tipos de fertilización en El Castillito, Las Sabanas, Madriz. Managua, Nicaragua: Facultad de Agronomía, Universidad Nacional Agraria.

Castañeda-Sánchez, A. (2011). Propiedades nutricionales y antioxidantes del maíz azul (Zea mays L.). Temas Selectos en Ingeniería de Alimentos, 5(2), 75-83.

Cortés, L., Hernández, J. M., Sangerman-Jarquín, D. M., \& Morán, S. H. (2013). Los maíces criollos y su conservación desde la perspectiva de los productores. Primer Congreso Nacional de Ciencia y Tecnología Agropecuaria (págs. 2637). Roque, Celaya, Guanajuato, México: Somecta.

Fernández, R., Morales, L. A., \& Gálvez, A. (2013). Importancia de los maíces nativos de México en la dieta nacional; una revisión indispensable. Revista Fitotecnia Mexicana, 36(Suplemento 3-A), 275-283. 
Guillén-Sánchez, J., Mori-Arismendi, S., \& Paucar-Menacho, L. M. (2014). Características y propiedades funcionales del maíz morado (Zea mays L.) var. subnigroviolaceo. Scientia Agropecuaria, 5(4), 211-217.

Hellin, J., Keleman, A., López, D., Donnet, L., \& Flores, D. (2013). La importancia de los nichos de mercado; un estudio de caso del maíz azul y del maíz para pozole en México. Revista Fitotecnia Mexicana, 36(Especial 6), 315-328.

Hernández-Martínez, V., Salinas-Moreno, Y., Ramírez-Díaz, J. L., Vázquez-Carrillo, G., Domínguez-López, A., \& RamírezRomero, A. G. (2016). Color, phenolic composition and antioxidant activity of blue tortillas from Mexican maize races. CYTA - Journal of Food, 14(3), 473-481. doi:10.1080 /19476337.2015.1136842

Ibarra, E. (2003). Las sociedades cacicales de Costa Rica (Siglo $X V I)$. San José, Costa Rica: Editorial UCR.

IBPGR. (1991). Descriptors for maize. Roma, Italia: International Board for Plant Genetic Resources.

Junior, B. B., Melo, A. E., Matias, J. N., \& Fontes, M. A. (2015). Avaliação de variedades crioulas de milho para produção orgânica no Semiárido Potiguar. Holos, 3, 102108. doi:10.15628/holos.2015.2277

López, M. A. (1997). Caracterización y evaluación preliminar de 33 cultivares de maíz (Zea mays L.) recolectados en diferentes localidades de Nicaragua. Managua, Nicaragua: Programa de Recursos Genéticos Nicaragüenses, Facultad de Agronomía, Universidad Nacional Agraria.

López-Romero, G., Santacruz-Varela, A., Muñoz-Orozco, A., Castillo-González, F., Córdova-Téllez, L., \& VaqueraHuerta, H. (2005). Caracterización morfológica de poblaciones nativas de maíz del Istmo de Tehuantepec, México. Interciencia, 30(5), 284-290.

López-Torres, B. J., Rendón-Medel, R., \& Camacho, T. C. (2016). La comercialización de los maíces de especialidad en México: condiciones actuales y perspectivas. Revista Mexicana de Ciencias Agrícolas(Publicación especial No. 15), 3075-3088.

Melgara, Y. d., \& Tinoco, O. D. (2013). Caracterización y evaluación preliminar de 32 accesiones de maíz (Zea mays L.) en Tisma, Masaya, postrera 2011. Managua, Nicaragua: Departamento de Producción Vegetal, Facultad de Agronomía, Universidad Nacional Agraria.

Mendoza, C. A., \& Gaitán, J. P. (2013). Caracterización y evaluación preliminar de 33 accesiones de maíz (Zea mays L.) colectadas en Nicaragua, Tisma, Masaya, postrera 2011. Managua, Nicaragua: Departamento de Producción Vegetal, Facultad de Agronomía, Universidad Nacional Agraria.

Ministerio de Cultura y Juventud. (2014). Maíz criollo integra alimentación y tradiciones por miles de años. Recuperado de http://www.mcj.go.cr/sala_prensa/noticias/2014/ agosto/noticias/consecutivo368.aspx

Montoya, F. (2011). Maize survives its makers in the Nicoya Peninsula. Terralingua Langscape, 2(9), 10-16.
Morales, D. (1993). Caracterización y evaluación preliminar de 21 genotipos de maíz (Zea mays L.). Managua, Nicaragua: Programa de Recursos Genéticos Nicaragüenses, Facultad de Agronomía, Universidad Nacional Agraria.

Olson, M. B., Morris, K. S., \& Méndez, E. (2012). Cultivation of maize landraces by small-scale shade coffee farmers in western El Salvador. Agricultural Systems, 111, 63-74. doi:10.1016/j.agsy.2012.05.005

Real Academia Española. (2017). Significado de la palabra pujagua. Recuperado de Diccionario de la Lengua Española; edición del tricentenario: http://dle.rae. es/?id=UbcVsWu

Ronceros, G., Ramos, W., Arroyo, J., Galarza, C., Gutiérrez, E. L., Ortega-Loayza, A. G., ... Palma, L. (2012). Estudio comparativo del maíz morado (Zea mays L.) y simvastatina en la reducción de lípidos séricos de pacientes diabéticos normotensos con dislipidemia. Anales de la Facultad de Medicina, 73(2), 113-117. doi:10.15381/anales.v73i2.859

Salinas-Moreno, Y., Pérez-Alonso, J. J., Vázquez-Carrillo, G., Aragón-Cuevas, F., \& Velázquez-Cardelas, G. A. (2012). Antocianinas y actividad antioxidante en maíces (Zea mays L.) de las razas Chalqueño, Elotes Cónicos y Bolita. Agrociencia, 46, 693-706.

Sánchez-Hernández, E., Cruz-Lázaro, E. d., \& SánchezHernández, R. (2015). Productividad y caracterización varietal de maíces nativos (Zea mays L.) colectados en Tabasco, México. Acta Agrícola y Pecuaria, 1(1), 7-15.

Sandri, C. A., \& Tofanelli, M. B. (2008). Milho crioulo: uma alternativa para rentabilidade no campo. Pesquisa Agropecuária Tropical, 38(1), 59-61.

Serna-Saldívar, S. O., Gutiérrez-Uribe, J. A., Mora-Rochín, S., \& García-Lara, S. (2013). Potencial nutracéutico de los maíces criollos y cambios durante el procesamiento tradicional y con extrusión. Revista Fitotecnia Mexicana, 36(Suplemento 3-A), 295-304.

Serratos, J. A. (2009). El origen y la diversidad del maíz en el continente americano. México: Greenpeace.

Sibaja, L. F., \& Zelaya, C. (2015). Nicoya: su pasado colonial y su anexión o agregación a Costa Rica. San José, Costa Rica: EUNED.

Uarrota, V. G., Severino, R. B., \& Maraschin, M. (2011). Maize landraces (Zea mays L.): a new prospective source for secondary metabolite production. International Journal of Agricultural Research, 6(3), 218-226. doi:10.3923/ ijar.2011.218.226

Uriarte-Aceves, P. M., Cuevas-Rodríguez, E. O., Gutiérrez-Dorado, R., Mora-Rochín, S., Reyes-Moreno, C., Puangpraphant, S., \& Milán-Carrillo, J. (2015). Physical, compositional, and wet-milling characteristics of Mexican blue maize (Zea mays L.) landrace. Cereal Chemistry, 92(5), 491-496. doi:10.1094/CCHEM-01-15-0001-R 